vations are based on relatively small numbers.

These studies represent the culmination of many years of research using human volunteers. The use of mentally handicapped children and prisoners in such studies has been criticized on many occasions and the background and justification for such studies were recently summarized by $S$. Krugman and J. P. Giles (J. Amer. Med. Ass., 212, 1019; 1970). Apart from the ethical issues involved, however, several questions are inevitably raised by these studies. First, it is known that although the MS-2 serum contains Australia antigen this serum gives rise to a mild and now well characterized form of serum hepatitis. Second, the agent of serum hepatitis has not been finally characterized and the injection of heated and diluted whole serum containing Australia antigen is rather a crude way of inducing immunity. The serum may, of course, be regarded as an inactivated "vaccine", but some purified inactivated vaccines such as measles and respiratory syncytial virus have sensitized rather than protected the recipients. In terms of hepatitis this is an important problem because several workers have suggested that there are similarities between Australia antigen-associated hepatitis and type 3 hypersensitivity reactions in which the severity of the disease may depend on the balance between antigen and antibody (see review by A. J. Zuckerman, Bull. Wld Hlth Org., 42, $957 ; 1970$ ). Finally, active immunization against serum hepatitis is likely to have only limited application, for example to children and adults in crowded institutions such as hospitals for the mentally handicapped and more so to certain groups at high risk to hepatitis such as patients and staff of renal dialysis units, transplant surgeons and perhaps some laboratory workers. The problem of active immunization against the much commoner epidemic form of hepatitis remains elusive.

\section{APHIDS}

\section{A Sticky End}

SOME species of wild potato seem to have a most effective mechanism for resisting attack by aphids-the glandular hairs on the leaves and stems discharge a gummy substance when damaged by the tarsi of an aphid; this exudate first of all restricts the insect's movements and further accumulations lead to the complete immobilization of the aphid and to its death. This mechanism is described by Dr R. W. Gibson of Long Ashton Research Station, Bristol, who has been experimenting with three species of wild potato, Solanum polyadenium, $S$. tarijense and $S$. berthaultii, and two aphid species, Myzus persicae and Macrosiphum euphorbiae (Ann. Appl. Biol., 68, 113; 1971).

In the three species of wild potato examined, the gummy exudate is produced from the tip of the glandular hair when an aphid mechanically ruptures the cell walls. In the intact hair, this substance seems to be a clear, water soluble liquid but on contact with air it changes to a dark brown or black insoluble sticky substance which is deposited on the aphid's limbs. Gibson found that not every contact of an aphid with a glandular hair resulted in the release of the exudate, but about 30 per cent of the aphids placed on any of the three species of wild potato became stuck to the plants by the substance within 24 hours. Starvation was probably the final cause of death; Gibson also noticed that the gummy substance occasionally found its way on to the labium of an aphid and this may also have prevented the aphids in his experiments from feeding.

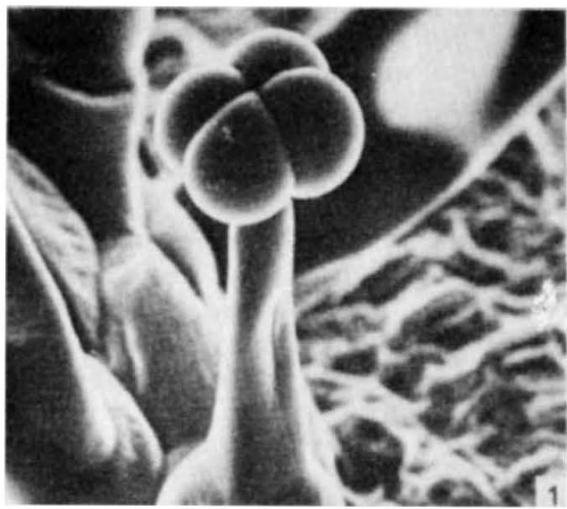

Glandular hair on Solanum berthaultii of the type consisting of a four-celled head mounted on a stalk.

Although Gibson observed a wide variety of hairs on the leaves and stems of the three wild potatoes, the type with the four-lobed head (see figure) mounted on a stalk seems to be the principal, if not the only, source of the gummy material. These hairs are abundant on

\title{
Tuming a Corner in Nucleic Acid Structural Studies
}

IN many ribonucleic acids, double helical base-paired regions are formed by a single polynucleotide chain folding back on itself. It is usually (and not unreasonably) assumed that these double helical regions have a structure similar to that determined by X-ray crystallography for the completely double helical molecules of reo virus and wound tumour virus RNA. But there have been no such impeccable guidelines for the conformation of the single stranded loops at the end of each double helix. Many of these loops, such as that containing the anticodon in transfer RNA, can be expected to have important roles in biological function and information on their structure is unquestionably of great significance. No doubt much will be revealed when the first single crystal analysis of a transfer RNA molecule is achieved. This happy event, however, still seems a little way off. In the interim attention has turned to the problem of turning a corner along a polynucleotide chain.

The information comes from the determination by Seeman et al. (see next Wednesday's Nature New Biology) of the crystal structure of the naturally occurring dinucleoside phosphate, uridine $3^{\prime}, 5^{\prime}$ adenosine phosphate (UpA). There are, in fact, two molecules of the dinucleotide in each asymmetric unit in this crystal, thus providing at some modest additional effort to the authors two independent determinations of the structure of UpA. Although these two molecules have quite different overall conforma- tions, Seeman et al. point out that these are attributable primarily to differences in orientation about only two single bonds-the two successive P-O bonds along the dinucleoside chain. The orientations found in one of the molecules are such that a polynucleotide chain containing a dinucleoside with such a conformation would turn a corner-actually through $180^{\circ}$ in the space of one phospho-diester link. This structure observed for UpA seems to represent a particularly neat solution for the production of a sharp bend in a polynucleotide chain and it would be surprising if use is not made of this stereochemical possibility of some close variant of it in studies of the biological function of nucleic acid.

The interbase hydrogen bonding in UpA is also of considerable interest. Although base pairing is common in crystals of bases, it is relatively rare in crystals of nucleosides and nucleotides where hydrogen bonding of the bases to water, sugar hydroxyls and charged phosphate oxygens is frequently observed. This is a point which many builders of transfer RNA models seem not to have realized, because in most models tertiary structure is attributed to base pairing between single stranded regions of the clover leaf. In this context the structure of UpA is an exception to the rule because it does have base pairing. This, however, is not complementary pairing in which adenine-uracil pairs of the WatsonCrick type hold together two molecules of UpA, but is self-pairing between uracils and also between adenines. 